\title{
An Algorithm for the semi-analytical Computation of Fields emitted in layered Ground by HVDC Electrodes
}

\author{
M. Brignone, A. Karimi Qombovani, P. Molfino, M. Nervi \\ Dept. of Electrical, Electronic, Telecommunications Engineering and Naval Architecture - (DITEN) \\ University of Genova \\ Genova, Italy \\ mario.nervi@unige.it
}

\begin{abstract}
For the design of HVDC ground electrodes it is of the utmost importance the availability of reliable and fast numerical tools, to evaluate both the fields and corrosion risk of nearby structures. Among the used algorithms, the Complex Images Method is well-known. However such method is often based on Prony's interpolator, which may show poor convergence properties, or even instability in particularly illconditioned cases. In this paper the use of a substitute interpolator, efficiently solving these drawbacks, named GPOF (Generalized Pencil Of Functions) is presented and results are discussed.
\end{abstract}

Index Terms-- Electromagnetic fields, Grounding, HVDC transmission, Interpolation, Numerical simulation

\section{INTRODUCTION}

The use of HVDC links is greatly spreading in recent years, as it shows a better behavior with respect to HVAC under several aspects, namely lower transmission cost and easier management when power has to be transmitted over long distance. All HVDC plants for their correct operation need power ground electrodes, that may be operated either continuously (leading to a configuration named monopolar plants) or just during faults (leading to a configuration named bipolar plants). Current flow through the multilayered ground may lead to direct safety issues (such as touch or step voltage exceeding the limits, directly depending on the dispersed current) and indirect safety issues (such as corrosion in nearby metallic structures, for example oil or methane pipelines, depending on the time integral of the dispersed current). Depending on these reasons, today the use of bipolar configuration is preferred, as it limits the duration of dispersion, and consequently reduces corrosion risk; furthermore, it allows the link to be operated at half power for a limited time during faults occurring to a converter. To estimate such adverse issues, particularly during the preliminary design phase, it is important to have very fast and uniformly accurate algorithm, taking correctly into account all boundary conditions, and modeling the interaction among the electrode and other metallic structures, which act as passive electrodes as well. In fact it is well-known that one of the most critical aspects of HVDC design is the correct selection of electrode site as, once that an unfit location has been selected, possible adverse effect like corrosion cannot be remedied by changing electrode shape and size. Another different feature that makes HVDC ground plants different from usual AC grounding systems is that in the latter case the study is particularly focused on an area of interest centered on the electrode. Unfortunately, while there are numerical algorithms that are perfect to model the field in a limited area (such as the Finite Element Method or the Finite Volume Method, which may be used to study the fields in the immediate vicinity of the electrode), the evaluation of distant effects could be performed much better by using semi analytical methods that do not discretize the space. In this way, we can obtain a reduction of processing time as well as much better accuracy in the areas where pipelines are buried. We considered the Method of Images, applying to the algorithm an interpolator, able to efficiently solve a problem that can arise adopting the commonly used approach.

\section{PROBLEM DESCRIPTION}

A number of significant simplifications had to be made to the problem to apply a semi analytical method, which is notoriously less flexible than a numerical method. Only electrodynamic stationary problems were studied, so inductances were neglected; even though a dynamic problem could in line of principle be studied, many commercial and well-tested analysis tools do exists, so our efforts were aimed at improving the analysis of HVDC grounds electrodes only. Furthermore, the ground was modeled as a horizontally layered ground. In line of principle, there is no limitation on the number of layers that can be efficiently analyzed. The electrode, as well as the pipelines, were modeled as a number of electrically connected cylinders of given radius (called the "segments"). This means that even a very complex geometry can easily be described as a 1D mesh, i.e. discretizing a number of line representing the axes of electrode trenches and of pipelines. This greatly simplifies the work of analyst and can easily be done using even public domain meshing software. For each section of electrode/pipe, the radius and the longitudinal electrical resistance has to be specified. It is also necessary to model how the different electrode sections are electrically connected to each other: as normally such connections are made using insulated cables, the topology of connecting network, together with the resistances of cables will have to be provided before the analysis. In this way a 
mixed field-circuit problem will be described, in such a way to comply with all the constraints and boundary conditions. The problem is current driven, so there will be a node, called "injection node", where the current is known. There can be several injection nodes within the same problem, to take into account possible interactions with other electrodes in the nearby, or to model distributed electrodes. To be remarked that no chemical polarization effect was considered, and that the ground is of course assumed to be composed by linear, time-invariant materials. The segments in our approach are not assumed to be horizontal; the only constraint is that a single segment may not cross the boundary between two layers. In this - unlikely- case, the segment must be split into two different sub-segments each one completely located within one single layer.

\section{THE METHOD OF IMAGES}

\section{A. The standard Method of Images}

The standard Method of Images has been largely used in the past; however, being based on infinite series, it is computationally inefficient and shows poor convergence properties, especially as the distance from the electrode increases, and field values are comparatively smaller [1-2]. Furthermore, the procedure for the generation of images may be quite tricky and time consuming. Normally the number of images to compute before the truncation is selected using an adaptive algorithm, which enforces the required accuracy: this may result in unpredictable computational times.

\section{B. The Method of Complex Images}

A substantial improvement of the standard Method of Images is the Method of Complex Images: it was initially introduced by Chow et al. for the computation of the electrostatic field within epitaxial semiconductors [3]. The major advantage of this method, straightforwardly applied by Chow also to current flow problems [4], is the very fast convergence, even using a low -and "a priori" fixed-number of images. However, even this method has a serious drawback, which is intrinsically due to the use of the so-called Prony's method: to build the approximate solution it is necessary to approximate along $\mathrm{Z}$ coordinate (i.e. the depth) a given number of sampling points $P(z)$, which depends on the layering and on boundary conditions. This must be made using as approximating functions a number $l$ of complex exponentials (called "the poles").

$$
P(z)=\sum_{j=1}^{l} \alpha_{j} e^{\beta_{j} z}
$$

The final result is the set of multiplicative coefficients of such exponentials $\alpha_{j}$, as well as the set of exponential coefficients $\beta_{j}$. Prony's method is able to determine them, with arbitrary precision. Sadly, Prony's interpolator is a two step algorithm based on exponentials, and it may show a rather unstable numerical behavior in many practical problems. As this algorithm practically is the key point of our field simulator, such behavior must absolutely be remedied.

\section{THE GPOF INTERPOLATOR}

The problem of approximation using complex exponentials arises in many practical problems; examining the relevant literature, we identified in the Generalized Pencil Of Functions (GPOF) a candidate algorithm to replace Prony's one. Such method is a single step algorithm normally used for the extraction of poles arising in high frequency problems [5], and particularly suitable when the number of coefficients is much lower than the number of samples to approximate. By simply using the GPOF as a substitute module for standard Prony's method, the overall behavior of the resulting algorithm greatly improves, retaining the fast convergence typical of the Method of Complex Images.

\section{COUPLING WITH A CIRCUIT SiMUlatoR}

The combination of the Complex Image Method based on the GPOF is sufficient to solve the electrodynamic field problem: based on the current dispersed by each dispersing segment, it is possible to compute the electric field in the ground. However such value is not known, as we normally just know the total dispersed current. To determine the distribution of dispersed current over each segment, in literature a technique was reported by Heppe [6], which initially determines all the self and mutual resistance coefficients among the segments. Afterwards it is imposed that the current shares over each segment according to such coefficients, and that the sum of all the dispersed current is the total electrode current, assuming that the electrode is equipotential (i.e. the voltage is constant over each segment). As a first approximation the hypothesis of equipotential electrode could be reasonable but, as the size of the electrode increases the difference of voltage over it may significantly impact on the current distribution. Therefore, to ensure accurate simulation, every segment (of the electrode, and of any other buried structure) was characterized by its longitudinal resistance, and a $\mathrm{T}$ equivalent circuit was identified. In other terms, half the longitudinal resistance of the segment was given to the each of the horizontal sides of the $T$, while the vertical side of the $T$ is the dispersing segment, defined using the already mentioned self and mutual resistance coefficients. In this way the problem may be casted through a block matrix, where one block is dense (as the field problem is approached using an integral approach), and a number of off-diagonal sparse blocks, deriving from the circuit coupling, which has been solved using the standard nodal analysis method. More precisely, if the electrode is discretized in $N_{\Sigma}$ segments, and $N$ are the physical nodes, $N_{1}$ the injection points and $N_{2}$ the connections between the injection point and the electrode, one has to solve the following linear system in $N_{\Sigma}+N+N_{1}+N_{2}$ unknowns:

$$
\left[\begin{array}{cccc}
\mathbf{Y}^{N N} & \mathbf{Y}^{N N_{\Sigma}} \mathbf{G} & \mathbf{Y}^{N N_{1}} & \mathbf{Y}^{N N_{2}} \\
\mathbf{Y}^{N N_{\Sigma}} & \mathbf{Y}^{N_{\Sigma} N_{\Sigma}} \mathbf{G}+\mathbf{1}_{N_{\Sigma}} & \mathbf{0} & \mathbf{0} \\
\mathbf{Y}^{N N_{1}} & \mathbf{0} & \mathbf{Y}^{N_{1} N_{1}} & \mathbf{Y}^{N_{1} N_{2}} \\
\mathbf{Y}^{N N_{2}} & \mathbf{0} & \mathbf{Y}^{N_{1} N_{2}} & \mathbf{Y}^{N_{2} N_{1}}
\end{array}\right]\left[\begin{array}{c}
\mathbf{U}^{N} \\
\mathbf{I}^{N_{\Sigma}} \\
\mathbf{U}^{N_{1}} \\
\mathbf{U}^{N_{2}}
\end{array}\right]=\left[\begin{array}{c}
\mathbf{0} \\
\mathbf{0} \\
\mathbf{J}^{N_{1}} \\
\mathbf{0}
\end{array}\right]
$$


Here, $\mathbf{U}^{\mathrm{X}}$ denotes the unknown potentials at nodes of kind $\mathrm{X}$, and $\mathbf{I}^{N_{\Sigma}}$ are the unknown currents flowing in the $N_{\Sigma}$ segments. $\mathbf{J}^{N_{1}}$ are the currents injected. As concern the coefficients matrix, $\mathbf{Y}^{\mathrm{AB}}$ are the block obtained analyzing the connections between nodes of kind A and B of the admittance matrix, and the Green matrix $\mathbf{G}$ whose entries are given by

$$
\mathbf{G}_{m n}=\frac{1}{L_{m}} \int_{P^{\prime} \in \Gamma_{m}} G\left(P_{n}, P^{\prime}\right) d s\left(\mathrm{P}^{\prime}\right)
$$

Here $\Gamma_{m}$ is the $m$-th segment of length $L_{m}$ in the discretization of the electrode and $P_{m}$ its middle point, while $G\left(P_{n}, P^{\prime}\right)$ is the Green function for observation point $P_{n}$ and source point $P^{\prime}$.

The only conceptual difference between electrodes and long buried metallic structures is that the total current dispersed by each electrode section is known and imposed, while the total current dispersed by each passive metallic structure is imposed to be zero. In this way we were able to properly compute the entering/exiting currents over each conducting segment taking into account all the relevant constraints. In any case, as the number of segments increases (i.e. the 1D meshing of conducting structures is refined), the accuracy of both the field and of the circuit simulation tends to improve.

\section{THE RESUlting AlgORITHM}

The resulting algorithm reads all the segment data (produced using a public domain 1D mesh generator) and all the other relevant data (layering definition, circuit connections data, et cetera). Afterwards the admittance matrix, the Green matrix and the injected currents are perfectly known, hence the equation system in (2) can be solved, in order to obtain as a result the values of current dispersed by each segment, and the voltage over each node of our circuit (and consequently the ground potential rise GPR). As final step, the requested fields (the voltage, and components/module of electric field and current density fields over a user-defined grid) are computed and stored into a database read by a public domain post-processor for the mapping of results.

\section{AlgORITHM TESTING AND RESUlTS}

The performance of the resulting algorithm was verified on a number of test problems available in technical literature, provided either with experimental measurements, or with numerical results. The test geometry, even though the algorithm is able to analyze any general electrode configuration, was assumed to be a ring, because this is the most common configuration, and many data are available in technical literature for comparison.

\section{A. Toroidal electrode in a horizontally multilayer soil}

According to the most typical soil structure [4], two case studies have been considered, in which a ring is buried in a horizontal multilayer soil structure (see Table I for details). All the basic grounding design quantities are calculated and reported as follow in Table II for all the different number of layers in both test cases 1 and 2 . The step voltage is defined as the voltage between two points located $1 \mathrm{~m}$ from each other. As the developed algorithm cannot manage electrodes with longitudinal resistance equal to zero, to simulate an ideal equipotential electrode, the reported initial computations were made assuming a longitudinal resistance equal to $10 \mu \Omega / \mathrm{m}$. With "different number of layers", we mean that the original 4 layers ground was modified to obtain simpler layerings, as follows. In particular, the upper layers (from the $1^{\text {st }}$ up to the $(\mathrm{i}-1)^{\text {th }}$ are defined as in the original definition, and the i-th one is extended to infinite depth. In Fig. 1 a 3D plot of the calculated surface voltage generated by the electrode defined in Case 1 is displayed.

TABLE I. ELECTRODE AND SOIL PARAMETERS

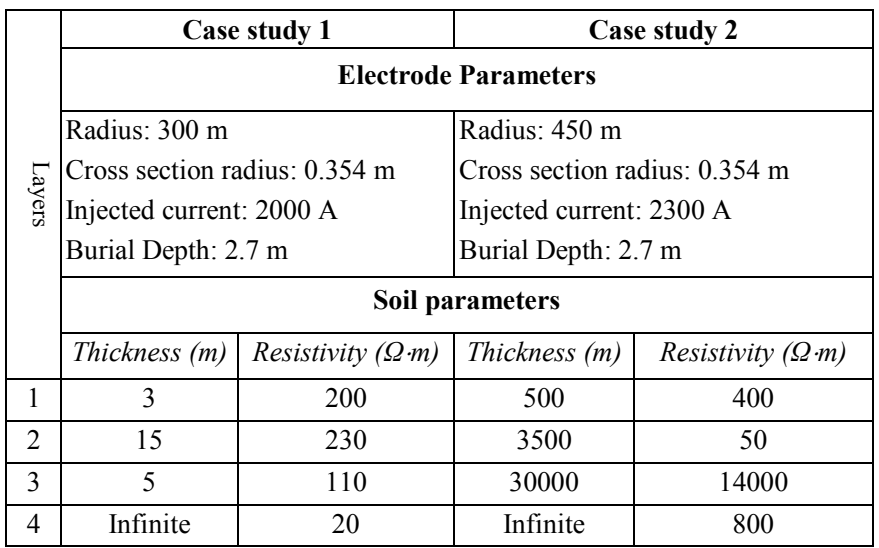

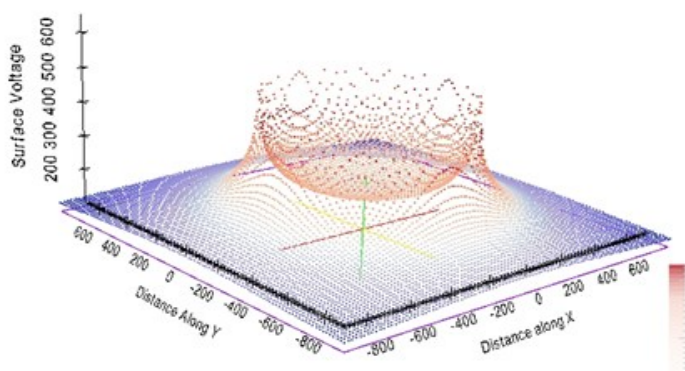

Figure 1. Earth Surface Voltage (ESV) profile for Case 1

TABLE II. GROUND MEASURED QUANTITIES

\begin{tabular}{|c|c|c|c|c|c|c|c|c|c|c|}
\hline \multirow{3}{*}{ 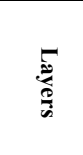 } & \multicolumn{5}{|c|}{ Case study 1} & \multicolumn{5}{|c|}{ Case study 2} \\
\hline & \multicolumn{5}{|c|}{ Maximum Values } & \multicolumn{5}{|c|}{ Maximum Values } \\
\hline & GPR & $\mathbf{R}_{\mathbf{G}}$ & $\begin{array}{l}\text { Current } \\
\text { Density }\end{array}$ & \begin{tabular}{|c|} 
Earth \\
Voltage
\end{tabular} & $\begin{array}{c}\text { Step } \\
\text { Voltage }\end{array}$ & GPR & $\mathbf{R}_{\mathrm{G}}$ & $\begin{array}{l}\text { Current } \\
\text { Density }\end{array}$ & \begin{tabular}{|c|} 
Earth \\
Voltage
\end{tabular} & $\begin{array}{c}\text { Step } \\
\text { Voltage }\end{array}$ \\
\hline 4 & 323 & 0.16 & 0.529 & 225 & 16.2 & 757 & 0.32 & 0.382 & 634 & 23.7 \\
\hline 3 & 465 & 0.23 & 0.518 & 367 & 17 & 812 & 0.35 & 0.372 & 690 & 23.7 \\
\hline 2 & 646 & 0.32 & 0.510 & 547 & 17.2 & 743 & 0.32 & 0.372 & 621 & 23.7 \\
\hline Homog. & 585 & 0.29 & 0.513 & 487 & 16.3 & 883 & 0.38 & 0.372 & 761 & 23.7 \\
\hline
\end{tabular}

According to the data in Table II, the grounding resistance $\left(R_{G}\right)$ and consequently the ground potential rise (GPR) show a considerable change depending on soil layering. On the contrary, the variations of current density and step voltage are 
affected by a much smaller difference. The results available in literature and our simulations confirm that a two layers soil structure seems to be the most difficult case to analyze with accuracy. In particular such observation is apparent in Fig. 2 and 3 .

\section{B. Deep layers influence}

When the distance between two ground electrodes in an HVDC system is large, a significant fraction of total current flows in the deep layers of the earth [4], affecting the potential distribution. The earth can be approximated as a multilayer sphere, each layer being characterized by a different resistivity value. As the distance between the electrodes used in a typical long distance link could be even $1000 \mathrm{~km}$ and the radius of the earth is more than $6000 \mathrm{~km}$, the arc length of the HVDC system on the earth's surface is short, so the earth can be studied as a horizontally stratified multilayer media. The ground normally does not present a uniform structure and layers are mostly horizontal, even though in some special cases they may be even vertical [2]. However, up to now, our approach can just analyze horizontal layers, even though we are evaluating the possibility to extend the algorithm to model also some vertical layer.

The Earth Surface Voltage (ESV) profiles on a radial path crossing the electrode trench have been calculated and plotted for different soil structures; in particular Fig. 2 reports the ESV profiles for the electrode as defined in Case 1 and Fig. 3 reports the ESV profile for the electrode as defined in Case 2.

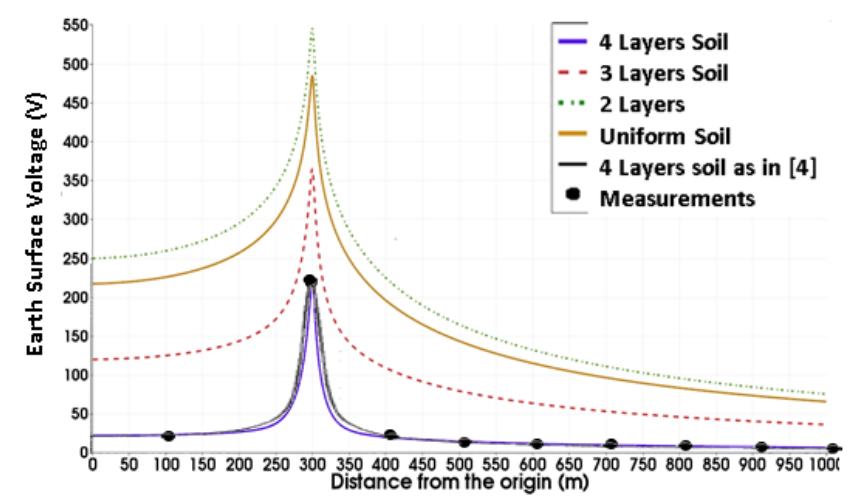

Figure 2. ESV profiles for different soil structures in Case 1.

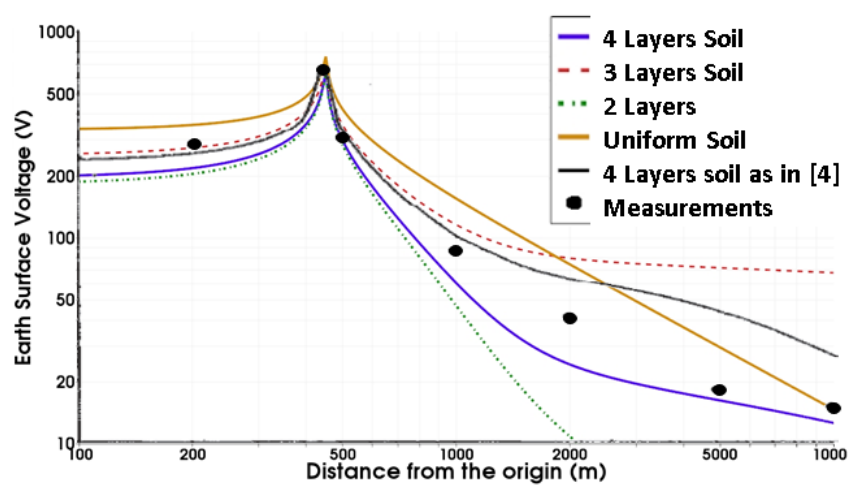

Figure 3. ESV profiles for different soil structures in Case 2.
The calculated ESV for Case 1 (Fig. 2) in a four layers soil grounding system is in good agreement with the measurements reported in [4]. The results are relatively closer to the reported ones especially in regions very far away from the electrode, where the influence of deep layers is more significant than that of the superficial ones. The accuracy in this region is of great importance for the study of interference with metallic installation buried in the vicinity of the electrode.

\section{Longitudinal resistance of the electrode segments}

In all the reported references about HVDC grounding systems, the influence of longitudinal resistance of the segments has not directly been taken into account. The proposed algorithm, on the contrary, may take into account all the resistances of the grounding station, starting from main feeding cables, distribution cables and also the longitudinal resistance of the segments in which the electrode is discretized. The dependence of ESV on the value of longitudinal segment resistance has been calculated and plotted for Case 2 in a four layers soil grounding system as reported in Fig. 4. To make the effect of the longitudinal resistance more evident, instead of feeding the toroidal electrode in a number of uniformly distributed points (as it is normally done in real electrodes), all calculations have been done considering just one injection point. With this uneven distribution, the difference with respect to an equipotential ideal electrode is greatly magnified. Of course the radial segment where the ESV is plotted is very close to the electrode's injection point.

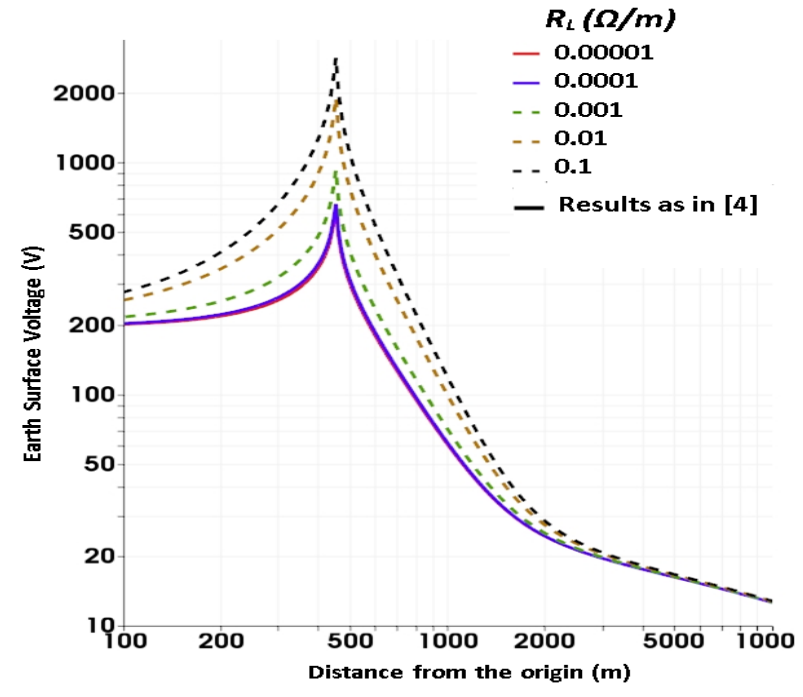

Figure 4. ESV profile for different longitudinal resistance in Case 2.

According to Fig. 4, for very low longitudinal resistances (between 10 and $100 \mu \Omega / \mathrm{m}$ ), i.e. nearly an equipotential grounding system, the corresponding voltage profiles are the closest to the results obtained for an ideal conductor as the one reported in [4]. If we compute the step voltage profile over the same above defined radial path, its value is heavily dominated by the longitudinal resistance value, as reported in Fig. 5. If step voltage is computed in points located more far away from the electrode than 2 to 3 times its radius (reported in Tab. 1), the effect is minimal. The computed step voltage 
shows an increase as the longitudinal resistance increases. Such a large variation highlights the key importance of a suitable network of distribution cables, so as the voltage and current distribution are characterized by a good uniformity over the electrode. The features that were introduced in the proposed algorithm make very easy to design a proper electrode distribution network.

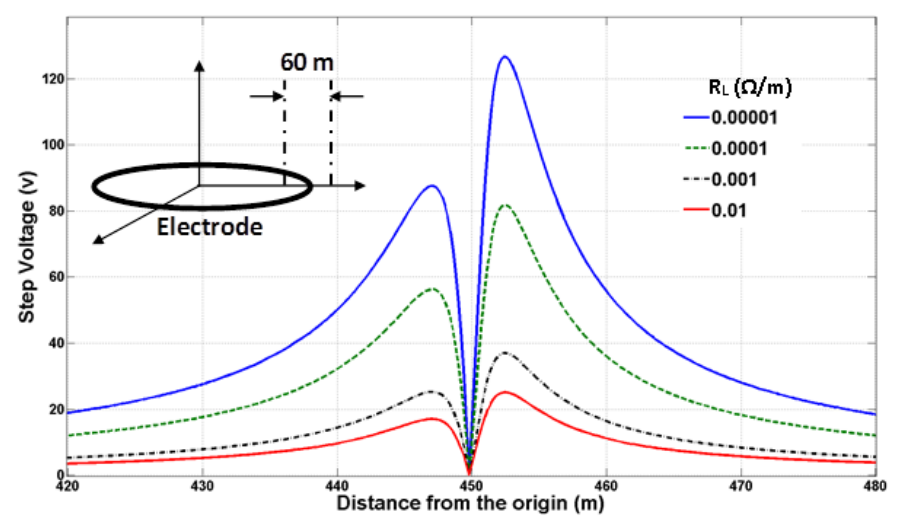

Figure 5. Step voltage profile for different longitudinal resistances in Case 2.

\section{Interference with underground metallic structures}

In order to study the interference between the grounding electrode and other underground metallic structured, an example reported in [7] has been considered. The buried structure is a $100 \mathrm{~km}$ long pipeline, which, in the closest point, is positioned $700 \mathrm{~m}$ far from the electrode center (Fig. 6). All the grounding parameters are the same as in Table I, Case 2, except for the injected current, set to $200 \mathrm{~A}$, and for the resistivity or the first layer, set to $150 \Omega \cdot \mathrm{m}$ according to [7], to make the comparison more reliable. The stray voltage (i.e. the voltage that the dispersed current flow causes on the affected infrastructure, w.r.t. a point located at infinite distance) along $50 \mathrm{~km}$ of the pipeline length, starting from the point with minimum distance from the electrode, have been calculated for different values of pipeline longitudinal resistance and it is reported in Fig. 6.

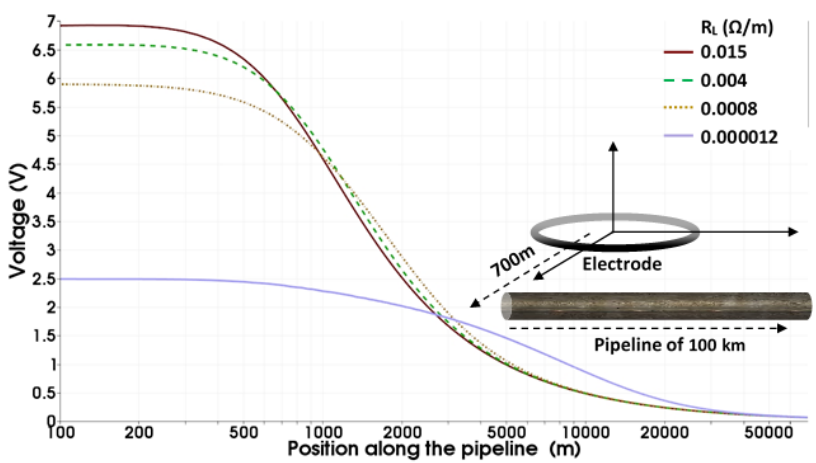

Figure 6. Stray Voltage on the pipeline and scheme of the experiment set-up.

The current density entering/exiting the pipeline is reported in Fig. 7. The induced current decreases as the resistance of segments in the pipeline increase. The "Cumulative Current", i.e. the current flowing through the pipeline is reported in Fig. 8 varies according to the same qualitative behavior.

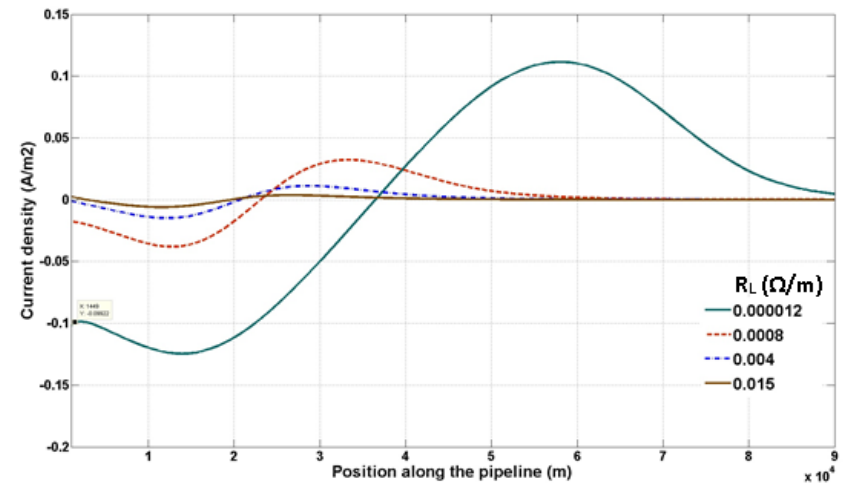

Figure 7. Current density entering/exiting the pipeline.

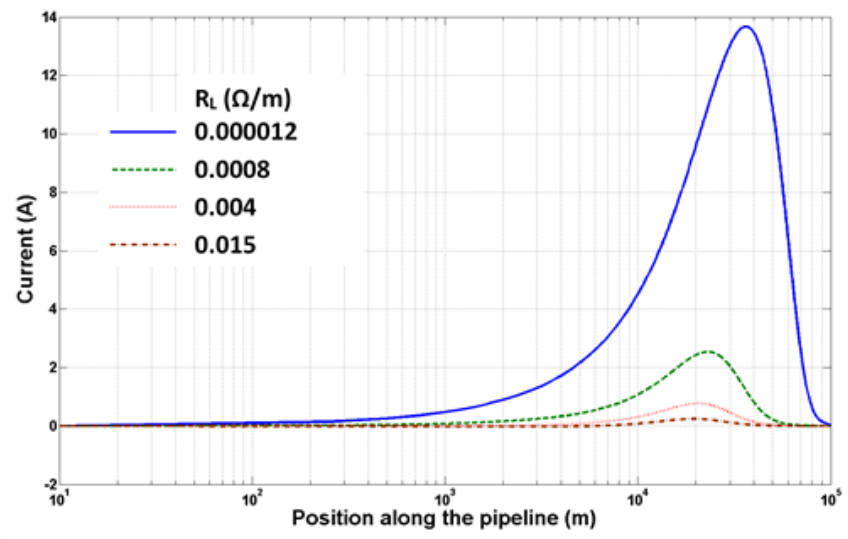

Figure 8. Cumulative current induced on the pipeline.

To verify the effect on the stray voltage caused by different layerings, computations have been done and the results are reported in Fig. 9. It is apparent that the structure of the soil can have a significant impact on Stray Voltage values.

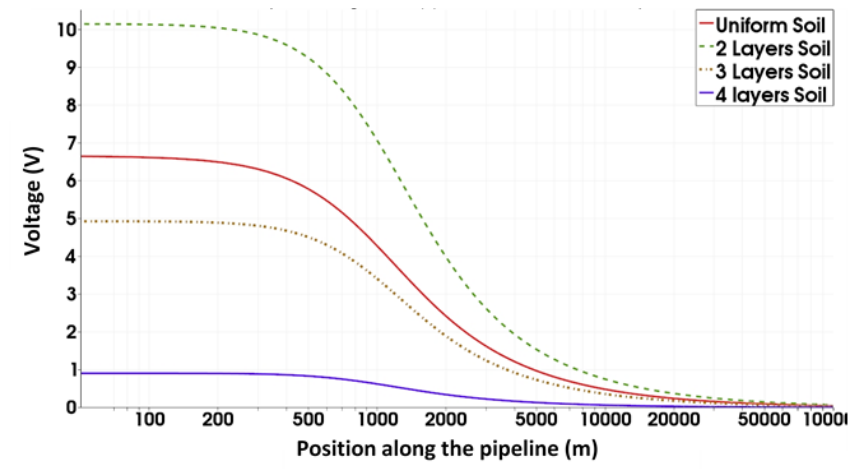

Figure 9. Stray voltage on the pipeline for different soil structure.

\section{DISCUSSION AND CONCLUSION}

The combination of the Complex Image Method based on the GPOF and of a circuit simulator based on the nodal analysis allows to solve a field-to-circuit coupled problem like the one arising in the analysis of HVDC ground electrodes. In this way, it is possible to take into account all field issues, together with the electrical connection of all the sections of the electrode, also considering the longitudinal electrical resistance of both the electrode and all the potentially 
impacted metallic structures. Results show good agreement with experimental measurements reported in literature. The proposed algorithm is a viable and reliable tool that can be used during HVDC ground electrode design located within a horizontally multilayered ground. The overall behavior of the proposed method results to be good in terms of accuracy and speed. Furthermore, it is quite stable even when the ground layering presents unfavorable characteristics (such as thin layers, huge differences is resistivity among the layers, et cetera). The computational speed is very high for the analysis section (i.e. the time required to determine the current dispersed by each segment). On the contrary, the computation of the values that are output to a post-processor is slightly more computationally demanding, as this basically is an integral method. In other terms, to determine the fields in one point, a summation on all the values produced by every dispersing segment must be carried out, resulting in a computational time that rises proportionally to the square of segment numbers. Much research has been carried out about methods for the calculation of maximum touch and step voltages. The application of an appropriate numerical method based on recent computer aid design obviates the need to such a wide diversity of methods for calculating the GPR, touch and step voltages in a grounding system with arbitrary geometry of the electrode for a multilayer soil of different structures. Two cases studies reported in [4] have been simulated using the code developed based of the proposed algorithm and the results have been validated with the measurements reported in references. The algorithm also enables to calculate the stray voltage and current on metallic objects such as pipelines, making possible to quantitatively evaluate the risk of potential problems caused by the current dispersed by the electrode, such as electrolytic corrosion. The applications, however, are not limited to corrosion assessment and electrode design, but may also be applied also to problems of interference with $\mathrm{AC}$ systems as, for example, described and studied within reference [8].

\section{REFERENCES}

[1] P. J. Lagace, J. L. Houle, Y. Gervais, and D. Mukhedkar, "Evaluation of the voltage distribution around toroidal HVDC ground electrodes in N-layer soils," IEEE Trans. Power Delivery, vol. 3, no. 4, pp. 15731579, Oct. 1988.

[2] J. E. Telles Villas, C. Medeiros Portela, "Calculation of Electric Field and Potential Distributions Into Soil and Air Media for a Ground Electrode of a HVDC System", IEEE Trans. On Power Delivery, vol. 18 , no. 3, pp. 867-873, July 2003.

[3] Y. L. Chow, J. J. Yang, G. E. Howard, "Complex Images for Electrostatic Field Computation in Multilayered Media", IEEE Trans. on Microwave Theory and Tech., vol. 39, no. 7, pp. 1120-1125, July 1991.

[4] Y. L. Chow, J. J. Yang, K. D. Srivastava, "Complex images of a ground electrode in layered soil", J. Appl. Psys., vol. 71, no. 2, pp. 569-574, Jan. 1992.

[5] Y. Hua, T. K. Sarkar, "Generalized Pencil-of-Function Method for Extracting Poles of an EM System from its Transient Response", IEEE Trans. on Antennas and Propagation, vol. 37, no. 2., pp. 229-234, Feb. 1989.

[6] R. J. Heppe, "Computation of Potential at Surface above an energized Grid or other Electrode, allowing for non-uniform Current Distribution," IEEE Trans. on Power Apparatus and Systems, vol. 98, no. 6, pp. 1978-1989, Nov./Dec. 1979.

[7] P. J. Lagace, J. L. Houle, H. Greiss, and D. Mukhedkar, "Computer Aided Evaluation of Pipeline Current near toroidal HVDC Ground Electrodes," IEEE Trans. Power Delivery, vol. 4, no. 1, pp. 216-222, Jan. 1989

[8] G. Mei, Y. Sun, and Y. Liu, "Simulation on DC Current Distribution in AC Power Grid under HVDC Ground-Return-Mode," $J$. Electromagnetic Analysis \& Applications, vol. 2, pp. 418-423, July. 2010. 